\title{
The importance of the within-trial interval in the superiority of the recall over anticipation method of paired-associate learning
}

\author{
MITCHELL G. BRIGELL and CHARLES P. THOMPSON \\ Kansas State University, Manhattan, Kansas 66506 \\ and \\ SAM C. BROWN \\ University of Missouri, Columbia, Missouri 65201
}

\begin{abstract}
The magnitude of the difference between the recall and anticipation methods of paired-associate learning was shown to increase as the length of the within-trial interval increased in the recall method. It was noted that only those studies which used short within-trial intervals fail to consistently show the recall method to be statistically superior to the anticipation method. Recall method learning rate was not affected when the within-trial interval was filled, suggesting that rehearsal is not responsible for the effect of interval. Alternate mechanisms were suggested.
\end{abstract}

In an analysis of paired-associate (PA) learning, Battig and Brackett (1961) compared the anticipation and recall methods of list presentation. Their results indicated that learning by the recall method was significantly more efficient than learning by the anticipation method. As a result of this study and an article by Battig (1965) emphasizing the many measurement problems inherent in the anticipation method, the recall method has become the preferred procedure in PA studies. However, the factors underlying the difference in learning rate between the recall and anticipation methods have not yet been experimentally determined. In fact, comparisons of these methods subsequent to the Battig and Brackett study have not always shown reliably faster learning with the recall method. While most studies surveyed by us report numerically superior recall performance ( 22 of 24 studies), the magnitude of the effect has varied widely, and only 10 of the 22 studies report statistically significant recall method learning superiority.

There have been several attempts to explain the variation in the magnitude of the recall method superiority. Initial investigations focused on the characteristics of the to-be-remembered lists and were unsuccessful (e.g., Cofer, Diamond, Olsen, Stein, \& Walker, 1967).

Recently, Battig (1973) has implicated the length of the interval between the study and test segments of recall method learning (i.e., the within-trial interval) in determining the magnitude of recall-anticipation learning differences. Battig cited two lines of evidence in support of his contention. First, was Izawa's (1971) finding that

This research was supported in part through Experimental Training Grant 5T01 MH 08359 to the first author. Requests for reprints should be sent to Mitchell G. Brigell, Department of Psychology, Kansas State University, Manhattan, Kansas 66506. lengthening the within-trial interval in the recall method facilitated learning while lengthening the between-trial interval in the anticipation method had no effect on learning. Thus, while both the within-trial interval in the recall method and the between-trial interval in the anticipation method are intervals between studying and testing, lengthening the former facilitates recall method learning while lengthening the latter has no effect on anticipation method learning. Although Izawa chose to interpret her results differently, they clearly suggest that manipulation of the within-trial interval in the recall method should effect the size of recall method superiority in a direct comparison with the ancitipation method. Second, Battig noted that all studies which have used substantial within-trial intervals (Battig \& Brackett, 1961; Battig \& Wu, 1965; Cofer et al., 1967) have reported large differences between recall and anticipation method learning. In contrast, studies using short within-trial intervals have not consistently found such differences.

The primary goal of this study was to directly measure the effect that lengthening of the within-trial interval in the recall method has on the magnitude of the recall-anticipation learning difference. Accordingly, recall groups with short $(3 \mathrm{sec})$ and long $(24 \mathrm{sec})$ within-trial intervals were compared with an anticipation group. Because rehearsal during the within-trial interval may be the critical factor in facilitating subsequent recall, an additional recall group was added in which the $24-\mathrm{sec}$ within-trial interval was filled with a rehearsal-preventing task.

\section{METHOD}

\section{Subjects}

Ninety subjects were drawn from the undergraduate student 


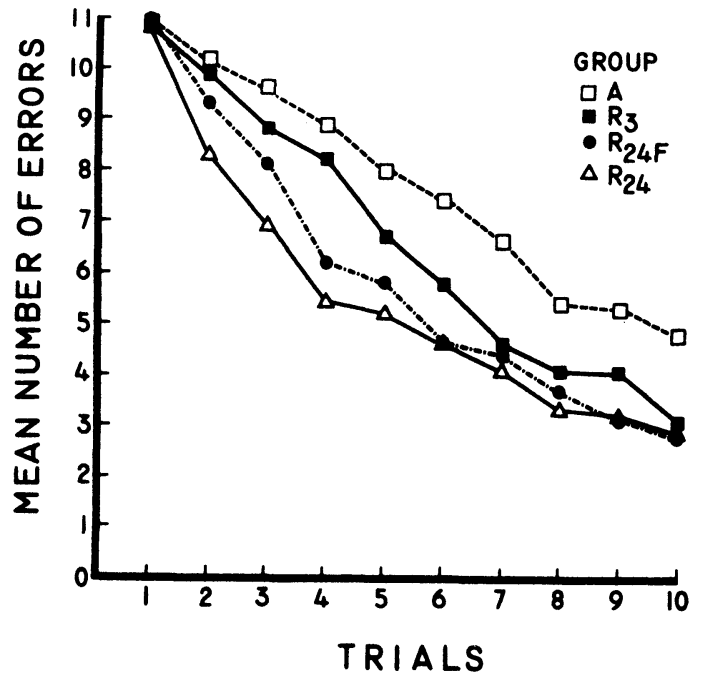

Figure 1. Mean number of errors as a function of trials for the four experimental groups.

population at Kansas State University. Some subjects received extra credit in their introductory psychology class, while others were paid for participating in the experiment. None of the subjects had participated in a prior PA study.

\section{Design}

The subjects were assigned randomly to one of four groups. One group of 36 subjects learned the 12-item list under the anticipation meth od of presentation (Group A). The three other groups of 18 subjects each were tested under the recall method with the characteristics of the within-trial interval different for each group. The subjects in Group $R_{3}$ had a 3-sec within-trial interval, while subjects in Group $R_{24}$ 'had a $24-s e c$ interval added between the study and test segments of a trial to distribute practice. Group $R_{24}$ f had a 24-sec within-trial interval filled with a shadowing task intended to limit opportunity for rehearsal.

\section{Materials}

The list consisted of 12 CVC-number pairs. The S-terms of the pairs were of medium association value $(\bar{X}=71 \%$; Archer, 1960) chosen for ease of pronounceability $(\overline{\mathrm{X}}=2.7$; Underwood \& Schulz, 1960). Two-digit numbers of low association value $(\overline{\mathrm{X}}=1.06$; Battig \& Spera, 1962) served as R terms. The $12 \mathrm{~S}$ and $12 \mathrm{R}$ terms were combined randomly to create the pairs. The list was presented visually on the wall in front of the subject by a slide projector. The shadowing task which filled the interval for Group $R_{24} f$, consisted of a list of two-syllable nouns, presented one per second auditorily by means of a tape recorder. A different random order of list presentations was used for each subject.

\section{Procedure}

Each subject, tested individually, learned the same 12-pair list to a criterion of two successive errorless trials or for a total of 10 trials, whichever occurred first. The subjects were instructed to vocalize the correct response during pair presentation and to respond verbally to the test stimuli if they thought they knew the response. Guessing was encouraged.

Stimulus presentation time was $3 \mathrm{sec}$, and all groups had a 6 -sec interval between trials. The within-trial interval was varied in the recall method groups as described in the design section. There is no within-trial interval possible in the anticipation method.

Since no learning measurement is possible on Trial 1 in the anticipation method, a Trial 0 preceded Trial 1 in both methods. This trial consisted of a normal anticipation trial in the anticipation condition and a presentation of the test stimuli alone in the recall conditions. Thus, testing on Trial 1 was preceded by one presentation of the test stimuli and one presentation of the pairs in both methods.

\section{RESULTS}

The number of errors to reach the criterion of two consecutive errorless trials or a maximum of 10 trials was tabulated separately for each subject on each trial. The criterion of two successive errorless trials was reached before Trial 10 by 15 of the 90 subjects with no subject reaching criterion in less than five trials. Figure 1 shows the mean number of errors made on each trial for the four groups. As can be seen, performance was virtually equivalent for all groups at the outset of learning (Trial 1) but quickly diverged as learning progressed. Overall, performance was best for Groups $\mathrm{R}_{24}(\overline{\mathrm{X}}=54.7$ errors $)$ and $\mathrm{R}_{24 \mathrm{f}}(\overline{\mathrm{X}}=59.2)$, poorest for Group A $(\overline{\mathrm{X}}=77.4)$ and intermediate for Group $\mathrm{R}_{3}$ $(\bar{X}=66.1)$. The analysis of variance showed a significant main effect of groups, $F(3,86)=3.24, p<.05$, a significant main effect of trials, $F(9,774)=256.76$, $\mathrm{p}<.01$, and a significant Groups by Trials interaction, $F(27,774)=2.76, p<.01$. A Newman-Keuls posttest performed on the mean number of errors per group revealed that except for the comparison between Groups $R_{24}$ and $R_{24}$, all pairwise comparisons were reliably different.

\section{DISCUSSION}

The results of this study clearly support the hypothesis that increasing the within-trial interval in the recall method of PA learning will increase the difference between recall and anticipation learning rates. The 24-sec within-trial interval produced reliably better recall than the $3-\mathrm{sec}$ interval. It is not particularly surprising, therefore, that studies which have used short within-trial intervals have not consistently shown the recall method to be statistically superior to the anticipation method. On the other hand, this is the fourth study comparing the methods which used a fairly large within-trial interval, and all four studies have found large and reliable differences in learning rates between the two methods.

It is not clear as yet how the within-trial interval serves to facilitate recall method performance. Rehearsal can probably be eliminated as the causal agent because the recall group which was tested after a filled interval $\left(R_{24} f\right)$ was not reliably different from the recall group which was tested after an unfilled interval $\left(R_{24}\right)$. Although it is possible to argue that activity during the filled interval did not eliminate rehearsal, it would seem very likely that the intervening activity would greatly reduce rehearsal. Failure to find a reliable difference between the two groups strongly suggests that rehearsal is not the critical mechanism. Another possibility is that subjects change their strategy for encoding the material as the within-trial interval increases. There is now developing considerable evidence that subjects change their strategy for recall as the conditions of retention change (Craik \& Watkins, 1973; Roenker, Note 1). Still another possibility is the temporal separation hypothesis proposed by Battig and Brackett (1961). Increasing the time 
during which no list-related task is performed could serve to further separate learning and testing into two distinct tasks and thereby facilitate performance. Unfortunately, that hypothesis is difficult to test without altering the methods of list presentation.

Although the present study was not designed to test either the differential encoding or separation hypotheses, both would seem to be plausible candidates for the mechanism underlying facilitation of recall through an extended within-trial interval.

In summary, the present experiment provides convincing evidence that lengthening the within-trial interval in the recall method increases the superiority of that procedure over the anticipation method of PA learning. Thus, it seems likely that the many failures reported in replicating Battig and Brackett's (1961) results can be attributed to the short within-trial intervals used in those studies. That is not to suggest that the magnitude of the within-trial interval is the only variable contributing to differences between recall and anticipation PA learning. Multiple causation for phenomena is the rule in psychology, and it would be very surprising if the differences between these two PA procedures proved to be an exception.

\section{REFERENCE NOTE}

1. Roenker, D. L. The role of rehearsal in long-term retention. Unpublished doctoral dissertation, Kansas State University, 1973.

\section{REFERENCES}

Archer, E. J. A re-evaluation of the meaningfulness of all possible CVC trigrams. Psychological Monographs, 1960, 74, (10, Whole No. 497).
Battig, W. F. Evidence that recall-anticipation differences increase with increasing time intervals. Journal of Experimental Psy chology, 1973, 100, 399-402.

Battig, W. F. Procedural problems in paired-associate research. Psy chonomic Monograph Sup plements, 1965, 1, No. 1 .

Battig, W. F., \& Brackett, H. R. Compariscn of anticipation and recall methods in paired-associate learning. Psychological Reports, 1961, 9, 59-65.

Battig, W. F., \& Spera, A. J. Rated association values of numbers from 0-100. Journal of Verbal Learning and Verbal Behavior, $1962,1,200-202$

Battig, W F \& Wu, R. D. Comparison of recall anticipation paired-associate procedures within mixed aurally presented lists. Psy chonomic Science, 1965, 3, 233-234.

Cofer, C. N., Diamond, F., Olsen, R. A., Stein, J. S., \& Walker, $H$. Comparison of anticipation and recall methods in paired-associate learning. Journal of Experimental Psychology, $1967,75,545-558$

Craik, F. I. M., \& Watkins, M. J. The role of rehearsal in short-term memory. Journal of Verbal Learning and Verbal Behavior, 1973, 12, 599-607.

Izawa, C. Massed and spaced practice in paired-associate learning: List versus item distributions. Journal of Experimental Psychology, 1971, 89, 10-21.

Tulving, E., \& Arbuckle, T. Y. Sources of intratrial interference in immediate recall of paired associates. Journal of Verbal Learning and Verbal Behavior, 1963, 1, 321-334.

Underwood, B. J., \& Schultz, R. W. Meaningfulness and verbal learning. Philadelphia: Lip pincott, 1960. 